\title{
The meteoroid environment of comet 9P/Tempel 1 and the Deep Impact spacecraft
}

\author{
J. Vaubaillon ${ }^{1}$, F. Colas ${ }^{1}$, and L. Jorda ${ }^{2}$ \\ 1 Institut de Mécanique Céleste et de Calcul des Éphémérides, Observatoire de Paris, 77 avenue Denfert-Rochereau, 75014 Paris, France \\ e-mail: vaubaill@imcce.fr \\ 2 Laboratoire d'Astrophysique de Marseille, Site Pereisc, 13376 Marseille Cedex 12, France
}

Received 27 September 2005 / Accepted 13 December 2005

\begin{abstract}
On the 4th of July 2005 the Deep Impact probe reached comet 9P/Tempel 1. The flyby spacecraft observed the collision from a distance of several hundred kilometres. Here we examine the meteoroid environment of the nucleus and present a method for computing the spatial density of particles, as well as the number of collisions any probe is supposed to endure. The model is calibrated from observations of the comet taken at the Pic-du-Midi observatory. In particular we measured $[A f \rho]=272 \pm 25 \mathrm{~cm}$, at $R h=1.579$ au and $\phi=29.4^{\circ}$. The data acquired by the Stardust spacecraft were also used. Several thousands of $100 \mu \mathrm{m}$-size meteoroids are expected to have collided with Deep Impact. Centimetersize particles were a threat to the probe and the upper limit of the number of collision computed here was higher than unity. However the results depend on the differential size population index $s$, which is taken to be constant. Preliminary results show that a few collisions were recorded but no $\mathrm{cm}$-size meteoroids were encountered. Interpreting this result as a change of $s$ with the meteoroid size, we derive $s>3.5$ for $\mathrm{cm}$-size and larger particles ejected by comet 9P/Tempel 1 . This can be caused by a fragmentation process and shows the relevance of any observation of large meteoroids ejected by cometary nuclei, as it provides constraints on $s$. This method can be used in preparation of any space mission to a comet.
\end{abstract}

Key words. meteors, meteoroids - comets: individual: 9P/Tempel 1

\section{Introduction}

Comet 9P/Tempel 1 was the target of the Deep Impact spacecraft. Many comets show a meteoroid stream in their vicinity (Ishiguro et al. 2003; Reach et al. 2000). Such a stream is essentially composed of large particles $(0.1 \mathrm{~mm}$ to $\mathrm{cm}$ size $)$, which follow the comet on its orbit (Sykes et al. 1986). These particles are known to have a low bulk density, and may be seen as "fluffy aggregates" (Babadzhanov 2002; Levasseur-Regourd 2004). Several space probes have sampled the dust environment of cometary nuclei (e.g. Stardust and 81P/Wild 2 in January 2004; see also Tuzzolino et al. 2004). The results reveal a large number of small particles (micron-sized). Meteoroids are hard to detect by optical telescopes, and are best seen in the infrared or when the Earth intercepts a stream (Reach et al. 2000; Vaubaillon \& Colas 2005; Vaubaillon et al. 2005b). In addition, meteoroids cannot be separated easily from the bright coma composed of the micron-sized dust particles that surround the nucleus when it approaches the Sun. However, there is no doubt that such particles are present in the vicinity of cometary nuclei and that they can be a threat to any spacecraft approaching them (see e.g. Landgraf et al. 2004). Any detection of them is of great interest, since it provides constraints on the differential size population index $s$ (see also Sects. 5 and 6).
In this paper, we examine the environment of comet 9P/Tempel 1 in order to predict the spatial density of meteoroids encountered by the Deep Impact spacecraft during its flyby as a function of the slope $s$ of the dust size distribution. The final aim of this paper is to constrain the value of $s$ taking the number of impacts on the spacecraft into account . On the 4th of July 2005 the Deep Impact impactor sucessfully reached the nucleus of this comet while the flyby spacecraft observed the event (A'Hearn et al. 2005). An outburst of activity of the comet was observed in the following hours from space and on Earth. Preliminary results of the mission showed that a few large meteoroids were encountered, but the detailed analysis of these impacts (in particular the derivation of the masses or sizes) has not been done yet. We do not consider here the contribution from very large particles $(>0.1 \mathrm{~m})$ in orbit around the nucleus (Richter \& Keller 1995), although Fulle (1997) has shown that the resulting cloud can have a diameter of several hundred kilometers.

We first present some observations of comet 9P/Tempel 1 in Sect. 2. These observations are used to compute the absolute density of meteoroids around the nucleus with a model described in Sect. 3. Results and consequences for the spacecraft are described in Sects. 4 and 5. 


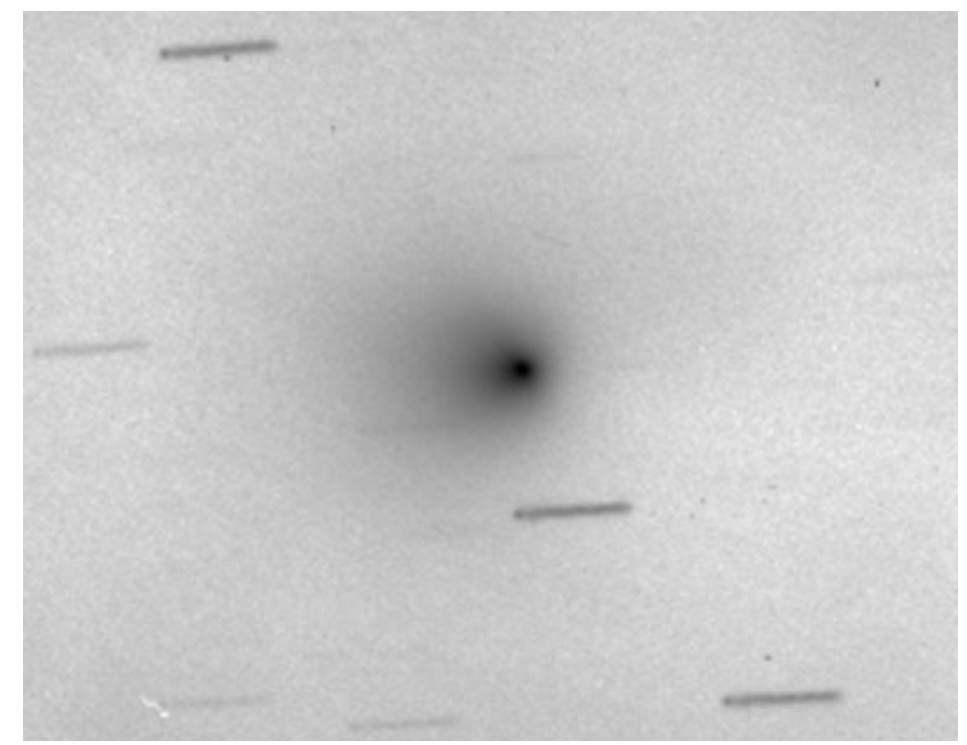

Fig. 1. Comet 9P/Tempel 1 observed with the 1 meter telescope located at Pic du Midi observatory (France), I filter, 1080 s. exposure time, on May 18th, $22 \mathrm{~h} 30 \mathrm{UT}, r_{\mathrm{h}}=1.579 \mathrm{AU}, \Delta=0.726 \mathrm{AU}$, Phase $\phi=29.4^{\circ}$.

Table 1. Physical parameters of comet 9P/Tempel 1, with $r_{\mathrm{n}}$ is taken from Lamy et al. (2001), $q$ from Rocher (see: www.imcce. $\mathrm{fr} / \mathrm{fr} /$ ephemerides/donnees/comets/FICH/CIFO0 18.php), [Af $\rho$ ] from Sect. 2, and $\alpha$ from Lara et al. (2005). $Q_{\mathrm{H}_{2} \mathrm{O}}(q)$ is from A'Hearn et al. (1995), and $f$ from Crifo \& Rodionov (1997), Appendix A, Eq. (9). $s$ is the differential size population index and is assumed (see also Sect. 4.2).

\begin{tabular}{ccc}
\hline \hline Symbol & Name & Value \\
\hline$r_{n}$ & radius of nucleus & $3.0 \mathrm{~km}$ \\
$q$ & perihelion distance & $1.51 \mathrm{AU}$ \\
{$[A f \rho]\left(r_{\mathrm{h}}=1.58 \mathrm{AU}, \phi=29.4^{\circ}\right)$} & {$[A f \rho]$ at $r_{\mathrm{h}}=1.58 \mathrm{AU}$} & $272 \pm 25 \mathrm{~cm}$ \\
$\alpha$ & index such as $\frac{[A f \rho]\left(r_{\mathrm{h}}\right)}{[A f \rho](q)}=\left(\frac{q}{r_{\mathrm{h}}}\right)^{\alpha}$ & 6.71 \\
{$[A f \rho]\left(q, \phi=29.4^{\circ}\right)$} & {$[A f \rho]$ at perihelion } & $373 \mathrm{~cm}$ \\
$Q_{\mathrm{H}_{2} \mathrm{O}}(q)$ & gas production rate & $1.90 \times 10^{28} \mathrm{mol.s} \mathrm{s}^{-1}$ \\
$f(q)$ & fraction of active area & 0.11 \\
$s$ & diff. size population index & 3.25 \\
\hline
\end{tabular}

\section{Observations}

Comet 9P/Tempel 1 has been monitored at the Pic du Midi observatory using the one meter telescope since October 2004. Our goal is to collect data taken in the same conditions before and after July 4, 2005 in order to have a clear idea of the influence of the impact on the dust coma. In this article, we only use the most recent data taken on May 18, 2005 with broadband $B$, $V, R$, and $I$ filters. For each filter, we co-added frames in order to create a composite image equivalent to an exposure time of 20 min (Fig. 1). We used Landolt (1992) photometric standard stars to perform the absolute calibration of these images.

The value of the $[A f \rho]$ parameter (A'Hearn et al. 1984) is calculated directly from the calibrated images. For a diaphragm of radius $\rho=15100 \mathrm{~km}$, we found $[A f \rho]=272 \pm 25 \mathrm{~cm}$ in the $I$ band and $[A f \rho]=261 \pm 26 \mathrm{~cm}$ in the $R$ band. Values obtained with the $B$ and $V$ broadband filters and with other diaphragms are fully consistent with these values. The $[A f \rho]$ values are significantly lower than the values measured by Lara et al. (2005), especially if one takes into account the reduction of the heliocentric distance of the comet from April 14 to May 18, 2005 (1.57 instead of $1.71 \mathrm{AU})$. The difference is at least partially due to a change of the phase angle, which increased from 13 degrees to about 30 degrees between these dates and also to dust production rate fluctuations.

\section{Model}

The model used in this approach is the one described by Vaubaillon et al. (2005a). It consists of a numerical simulation of the generation and the evolution of a meteoroid stream ejected by a comet. The creation of a stream is modeled by the ejection of meteoroids from the nucleus, according to the model developed by Crifo \& Rodionov (1997). The physical parameters of comet 9P/Tempel 1 used in this paper are summarized in Table 1 . The size distribution is approximated by a power law such as the one that says the number of particles of size $a$ is proportional to $a^{-s}$, with $s$ the differential size population index. For the evolution of the stream, the gravitation of the nine planets are taken into account, along with the nongravitational forces.

We consider five bins of size from $10^{-4}$ to $10^{-1} \mathrm{~m}$. As we wanted to compute the spatial density of meteoroids around the nucleus, we simulated both young and old meteoroid trails. As 

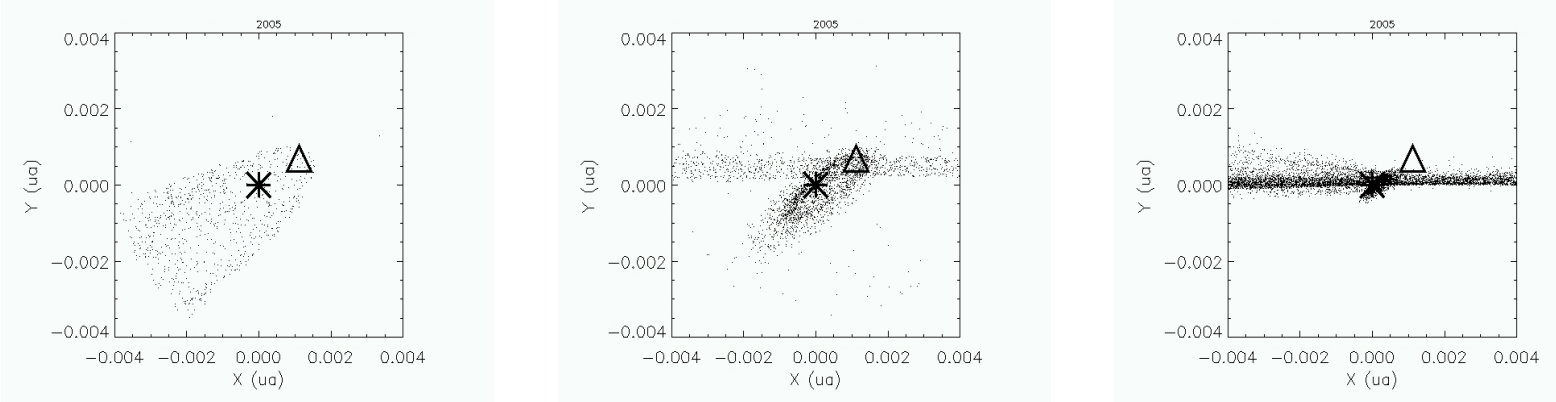

Fig. 2. Configuration of the meteoroid stream ejected by comet 9P/Tempel 1 (symbolized by the star) and the Deep Impact spacecraft (Triangle). Coordinates are $(X, Y)$ ecliptic, relative to the nucleus and rotated so that the $X$ axis points toward the direction of motion of the comet. Right: meteoroids of radius in the range $[0.1 ; 0.5] \mathrm{mm}$, center: $[1 ; 5] \mathrm{mm}$, left: $[10 ; 100] \mathrm{mm}$. Because of the combined effect of the ejection velocity and non-gravitational forces, the smallest particles are the youngest ones (ejected during the 2005 perihelion return only). The largest ones were ejected between 1937 and 2005.

Table 2. Physical parameters of comet 81P/Wild 2, with $r_{\mathrm{n}}$ is taken from Tsou et al. (2004), $q$ from Rocher (see: www.imcce.fr/fr/ephemerides/donnees/comets/FICH/CIF0008.php), $[A f \rho](q)$ from Farnham \& Schleicher (2005), $\alpha$ and $Q_{\mathrm{H}_{2} \mathrm{O}}(q)$ are from A'Hearn et al. (1995), and $f$ from Crifo \& Rodionov (1997), Appendix A, Eq. (9). $s$ is assumed (see also Sect. 4.2).

\begin{tabular}{ccc}
\hline \hline Symbol & Name & Value \\
\hline$r_{\mathrm{n}}$ & radius of nucleus & $2.5 \mathrm{~km}$ \\
$q$ & perihelion distance & $1.58 \mathrm{AU}$ \\
$\alpha$ & index such as $\frac{[A f \rho]\left(r_{\mathrm{h}}\right)}{[A f \rho](q)}=\left(\frac{q}{r_{\mathrm{h}}}\right)^{\alpha}$ & 1.68 \\
{$[A f \rho]\left(q, \phi=39.4^{\circ}\right)$} & {$[A f \rho]$ at perihelion } & $275 \mathrm{~cm}$ \\
$Q_{\mathrm{H}_{2} \mathrm{O}(q)}$ & gas production rate & $1.32 \times 10^{28} \mathrm{~mol} . \mathrm{s}^{-1}$ \\
$f(q)$ & fraction of active area & 0.103 \\
$s$ & size distribution index & 3.25 \\
\hline
\end{tabular}

we did not know how long large meteoroids can remain in the vicinity of the nucleus, we considered twenty-seven perihelion returns (the first in 1850 and the last in 2005). It appeared (see Sect. 4) that this is enough for our purpose. Indeed the oldest meteoroids still in the vicinity of the nucleus at the time of the encounter with the Deep Impact spacecraft are ejected in 1937 . For each bin of size, $5 \times 10^{4}$ particles were simulated, for a total of $6.75 \times 10^{6}$ particles. The simulations were run on 5 to 50 parallel processors of an IBM-SP4, located at CINES (France). A statistical weight is set to each simulated particle. This weight represents the real amount of meteoroids having a radius $a$ ejected by the comet. It is computed from the parameters of Table 1 with a method described in detail by Vaubaillon et al. (2005a). The orbit of each meteoroid is propagated until the day of the encounter (4th of July 2005, 0:00 UT). The youngest meteoroids considered here were ejected the day of the impact. The reason is that in the numerical model the meteoroids are ejected at each Julian day at 0:00 UT. The computation of the statistical weights takes this feature into consideration. The spatial density of meteoroids around the nucleus is then computed.

\section{Results}

\subsection{General context}

Figure 2 shows the position of the meteoroid stream relative to the comet nucleus. The ephemeris of the spacecraft was taken from the JPL HORIZONS ephemeris web site (http://ssd.jpl.nasa.gov/). In the figure, the spacecraft seems to be very far from the nucleus $(0.001 \mathrm{AU})$ because at the time of the computation of the ephemeris, it was still on a preliminary orbit that would lead it to the nucleus. This orbit was modified during the approach to the comet but the relative velocity remained unchanged.

In order to calibrate the model we used the data acquired by the Stardust spacecraft when passing by comet 81P/Wild 2 . Basically, we tried to reproduce the impact of 40 particles of radius $>100 \mu \mathrm{m}$ (only data before $+600 s$ were considered; see Tuzzolino et al. 2004). Table 2 sums up the features of comet 81P/Wild 2 used here. Considering the differences of physical parameters between the two comets and between the circumstances of the two encounters (see Table 3), the expected number of collisions between the Deep Impact spacecraft and $100 \mu \mathrm{m}$ size particles is $1.6 \times 10^{4}$. In order to consider the whole meteoroid stream, we also computed the density of particles using a numerical simulation.

\subsection{Spatial density of meteoroids}

The meteoroid spatial density around comet 9P/Tempel 1 is provided in Table 4. Since the spacecraft will pass within $500 \mathrm{~km}$ of the comet and because of the uncertainties in the orbit of the spacecraft at the time of the computation, the spatial density was computed by assuming that the spacecraft will pass through the nucleus. Thus the spatial densities provided in Table 4 are the maximum the flyby spacecraft can encounter. 
Table 3. Difference between the 2 encounters of Stardust with 81P/Wild 2 and Deep Impact with 9P/Tempel 1. A: surface of detector device (Stardust) or equivalent surface of the spacecraft during the encounter (Deep Impact, i.e. $1.7 \times 2.3 \mathrm{~m}^{2}$ since the spacecraft was rotated during the encounter); $V_{\text {rel }}$ : initial relative velocity between the spacecraft and the cometary nucleus; $D$ : minimum distance between the nucleus and the spacecraft; $N_{i}(a)$ : measured (Stardust, see Tuzzolino et al. 2003) or expected (Deep Impact) number of impacts with particles of size $a(\mu \mathrm{m})$.

\begin{tabular}{ccc}
\hline \hline Name & Stardust & Deep Impact \\
\hline$A\left(\mathrm{~m}^{2}\right)$ & $2.2 \times 10^{-2}$ & 3.91 \\
$V_{\text {rel }}\left(\mathrm{km} \mathrm{s}^{-1}\right)$ & 6.1 & 10.3 \\
$D(\mathrm{~km})$ & 236 & $\simeq 500$ \\
$N_{i}(3)$ & 1709 & $6.84 \times 10^{5}$ \\
$N_{i}(100)$ & 40 & $16 \times 10^{3}$ \\
\hline
\end{tabular}

The spatial density is computed in a region where the number of simulated particles is large enough for the computation to make sense. We consider that 100 particles is a minimum. Thus it was computed in a region of radius $2 \times 10^{-5} \mathrm{AU}=$ $3000 \mathrm{~km}$. The spacecraft passes through such a region in approximatively $290 \mathrm{~s}$ (at initial relative velocity).

Vaubaillon et al. (2005b) show that the differential size population index $s$ is a sensitive parameter when computing the statistical weights. It will then influence the derived spatial density of meteoroids. Figure 3 shows the influence of $s$ on the number of expected impacts for particles in the range $[0.1 ; 0.5] \mathrm{mm}$. For reasonable values of $s$ (i.e. in the range $[2.75 ; 3.75] \mathrm{mm}$ ), the expected number of impacts varies by a factor of 3 . In Table 4 we consider $s=3.25$. This value gives the maximum number of expected impacts.

\section{Discussion}

\subsection{Consequences for the flyby spacecraft}

Our model (see Table 4) predicts that the spacecraft would have encountered several thousand $100 \mu \mathrm{m}$ particles at its closest approach. It also shows that the largest particles that are likely to be encountered have a $10 \mathrm{~cm}$ radius. The Deep Impact flyby spacecraft was equipped with meteoroid shields that protected the instruments during the closest approach to the nucleus. The orientation of the flyby spacecraft was also changed in order to present the minium area to the meteoroid stream. This amount of protection is generally efficient for small meteoroids, but large ones (cm-size) are still dangerous for the spacecraft.

Because of the choice of the differential size population index $s$ (considered as constant on the whole size range), the results in Table 4 are an upper limit for what could be encountered. As seen in Sect. 4.2, the meteoroid spatial density can vary by a factor of three at least, depending on the value of $s$. But even with such a correction, the expected number of impacts with a cm-size meteoroid is almost one.

For this reason we conclude that an encounter with a cmsize meteoroid is not guaranteed, but is likely to happen. It was well known anyway that approaching a cometary nucleus involves certain risks for the spacecraft, and special care was

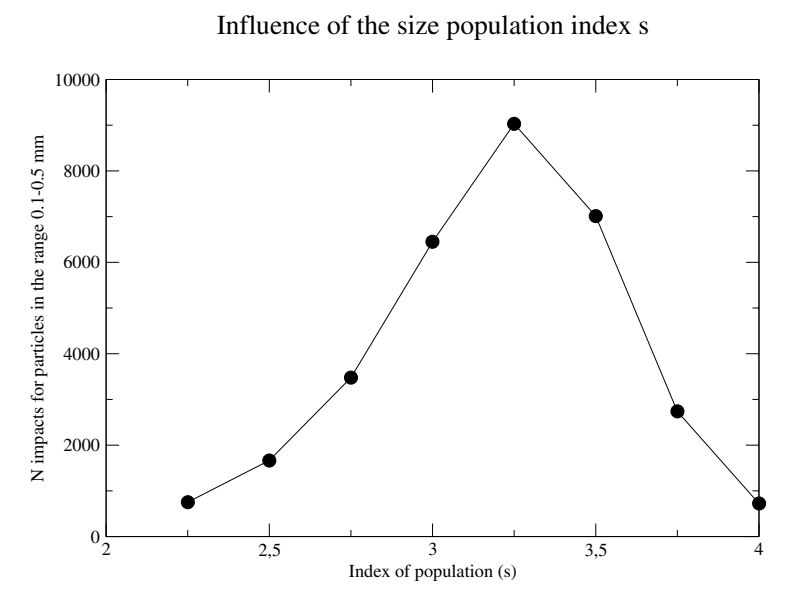

Fig. 3. Influence of the differential size population index $s$ on the expected numbers of impacts for particles in the size range $[0.1 ; 0.5] \mathrm{mm}$.

taken by the Deep Impact team during the closest approach to the nucleus. In particular the control of the orientation in such a case is important. We recall that the ESA Olympus satellite was lost following an impact with a Perseid meteoroid and false manoeuvers from the ground control (Caswell et al. 1995).

\subsection{Update from preliminary results of the mission}

Two impacts with large meteoroids were recorded on the impactor (A'Hearn et al. 2005), but it is impossible to make a statement on the size or mass of these objects right now. No large impact was recorded by the flyby spacecraft. These results confirm the presence of large particles in the vicinity of the nucleus. We have seen that the results presented in Table 4 are the maximum of the possible number of impacts, considering the physical properties of the comet and the circumstances of the encounter. The most sensitive parameter here is $s$, the differential size population index. Lisse C. (personal communication) suggested to us that in case of $9 \mathrm{P} /$ Tempel 1 , it would be closer to 2.8 rather than 3.25. Sugita et al. (2005) instead find a value of $3.5-3.6$ for particles up to $10 \mu \mathrm{m}$ size. In the case of $81 \mathrm{P} /$ Wild 2, this parameter varies with the size of the particles and depends on the region around the nucleus (inside or outside the swarm). Green et al. (2004) find that the cumulative index ranges from 0.3 to 1.1 , corresponding to a differential size population index of 1.9 to 4.3 . Considering only this latter value and assuming it is constant on the whole size range, the expected number of impacts would decrease by a factor of 30. However, such a computation is not physically realistic. Finally, the fragmentation process revealed by Green et al. (2004) and Levasseur-Regourd (2004) was not taken into account here. Harker et al. (2005) find that the dust emitted by the comet is fractal and porous $(D>2.8)$ making any fragmentation process likely. All these factors will cause the estimated number of impacts to decrease and explains the absence of a $\mathrm{cm}$-size impact on the flyby spacecraft. 
Table 4. Spatial density of meteoroids around the nucleus of comet 9P/Tempel 1, computed in a region centered on the cometary nucleus and of radius $2 \times 10^{-5} \mathrm{AU}$. The spacecrafts will stay for $290 \mathrm{~s}$ in such a region. $N_{\mathrm{s}}$ : number of simulated particles within $r_{\mathrm{d}}$ AU from the nucleus; $D$ : spatial density of particles; $N_{\mathrm{c}}$ : number of expected collisions for the flyby spacecraft.

\begin{tabular}{cccccc}
\hline \hline Size bin $(\mathrm{mm})$ & {$[0.1 ; 0.5]$} & {$[0.5 ; 1]$} & {$[1 ; 5]$} & {$[5 ; 10]$} & {$[10 ; 100]$} \\
\hline$N_{\mathrm{s}}$ & 200 & 313 & 530 & 790 & 2130 \\
$D\left(\mathrm{~km}^{-3}\right)$ & 7701 & 261 & 114 & 3.69 & 2.44 \\
$N_{\mathrm{c}}$ & 9033 & 306 & 134 & 4.33 & 2.88 \\
\hline
\end{tabular}

\section{Conclusion}

Comet 9P/Tempel 1, the target of the Deep Impact spacecraft, was observed at the Pic du Midi observatory before the encounter and is still being monitored these days. From the images taken we measured $[A f \rho]=272 \mathrm{~cm}$ at $r_{\mathrm{h}}=1.579 \mathrm{au}$ and $\phi=29.4^{\circ}$, and we computed the meteoroid spatial density around the nucleus and the number of impacts with a spacecraft. Several thousand impacts with $100 \mu \mathrm{m}$-size meteoroids were derived. As the population of large particles is poorly known, any detection improves the constraints on the value of $s$ a lot. The upper limit of the number of collision between $\mathrm{cm}$ size meteoroids and the Deep Impact spacecraft is higher than unity. This value depends on the value of $s$, considered to be constant in this approach. Preliminary results of the mission reveal that if impacts were recorded, no $\mathrm{cm}$-sizes were detected. Interpreting this as a change of $s$ with the meteoroid size, we derive $s>3.5$ for $\mathrm{cm}$-size and larger meteoroids. This variation can be the consequence of the fragmentation process reported by Levasseur-Regourd (2004).

This method can be used in the preparation phase of any space mission to a comet.

Acknowledgements. We are thankful to M. A'Hearn, P.I. of the Deep Impact mission for his advice, as well as to A. C. Levasseur-Regourd, D. Wellnitz, and C. Lisse. We thank the CINES team for their help and the use of the supercomputer.

\section{References}

A'Hearn, M. F., Schleicher, D. G., Millis, R. L., Feldman, P. D., \& Thompson, D. T. 1984, AJ, 89, 579

A'Hearn, M. F., Millis, R. L., Schleicher, D. G., Osip, D. J., \& Birch, P. V. 1995, Icarus, 118, 223
A’Hearn, M. F., Belton, M. J. S., Delamere, W. A., et al. 2005, Science, 310,258

Babadzhanov, P. B. 2002, A\&A, 384, 317

Caswell, R. D., McBride, N., \& Taylor, A. D. 1995, Inter. J. Imp. Eng., 17,139

Crifo, J. F., \& Rodionov, A. V. 1997, Icarus, 127, 319

Farnham, T. L., \& Schleicher, D. G. 2005, Icarus, 173, 533

Fulle, M. 1997, A\&A, 325, 1237

Green, S. F., McDonnell, J. A. M., McBride, N., et al. 2004, J. Geophys. Res. (Planets), 109, 12

Harker, D. E., Woodward, C. E., \& Wooden, D. H. 2005, Science, 310, 278

Ishiguro, M., Kwon, S. M., Sarugaku, Y., et al. 2003, ApJ, 589, L101

Lamy, P. L., Toth, I., A'Hearn, M. F., Weaver, H. A., \& Weissman, P. R. 2001, Icarus, 154, 337

Landgraf, M., Jehn, R., Flury, W., \& Dikarev, V. 2004, Adv. Space Res., 33, 1507

Landolt, A. U. 1992, AJ, 104, 372

Lara, L., Boehnhardt, H., Gredel, R., et al. 2005, IAU Circ., 8532, 3

Levasseur-Regourd, A. 2004, Science, 304, 1762

Reach, W. T., Sykes, M. V., Lien, D., \& Davies, J. K. 2000, Icarus, 148,80

Richter, K., \& Keller, H. U. 1995, Icarus, 114, 355

Sugita, S., Ootsubo, T., Kadono, T., et al. 2005, Science, 310, 274

Sykes, M. V., Lebofsky, L. A., Hunten, D. M., \& Low, F. 1986, Science, 232, 1115

Tsou, P., Brownlee, D. E., Anderson, J. D., et al. 2004, J. Geophys. Res. (Planets), 109, 12

Tuzzolino, A. J., Economou, T. E., Clark, B. C., et al. 2004, Science, 304, 1776

Tuzzolino, A. J., Economou, T. E., McKibben, R. B., et al. 2003, J. Geophys. Res. (Planets), 5

Vaubaillon, J., \& Colas, F. 2005, A\&A, 431, 1139

Vaubaillon, J., Colas, F., \& Jorda, L. 2005a, A\&A, 439, 751

Vaubaillon, J., Colas, F., \& Jorda, L. 2005b, A\&A, 439, 761 